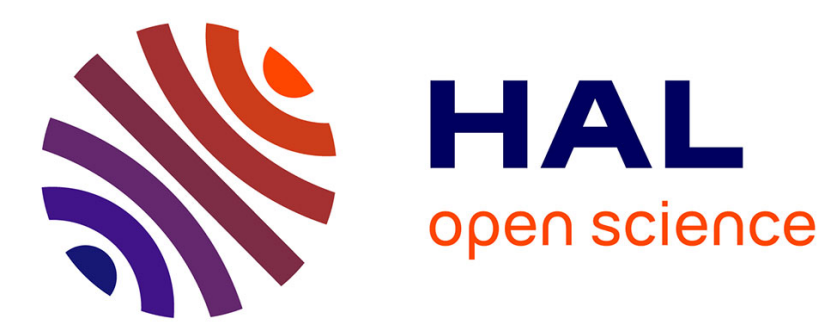

\title{
How rich is the class of processes which are infinitely divisible with respect to time?
}

Khalifa Es-Sebaiy, Youssef Ouknine

\section{To cite this version:}

Khalifa Es-Sebaiy, Youssef Ouknine. How rich is the class of processes which are infinitely divisible with respect to time?. Statistics and Probability Letters, 2007, 78, pp.537-547. hal-00363616

\section{HAL Id: hal-00363616 https://hal.science/hal-00363616}

Submitted on 12 May 2009

HAL is a multi-disciplinary open access archive for the deposit and dissemination of scientific research documents, whether they are published or not. The documents may come from teaching and research institutions in France or abroad, or from public or private research centers.
L'archive ouverte pluridisciplinaire HAL, est destinée au dépôt et à la diffusion de documents scientifiques de niveau recherche, publiés ou non, émanant des établissements d'enseignement et de recherche français ou étrangers, des laboratoires publics ou privés. 


\title{
How rich is the class of processes which are infinitely divisible with respect to time?
}

\author{
Khalifa ES-SEBAIY * and Youssef OUKNINE ** \\ $\left({ }^{*}\right)(* *)$ Department of Mathematics, Faculty of Sciences Semlalia, \\ Cadi Ayyad University 2390 Marrakesh, Morocco.
}

October 7, 2007

\begin{abstract}
We give a link between stochastic processes which are infinitely divisible with respect to time (IDT) and Lévy processes. We investigate the connection between the selfsimilarity and the strict stability for IDT processes. We also consider a subordination of a Lévy process by an increasing IDT process. We introduce a notion of multiparameter IDT stochastic processes, extending the one studied by Mansuy [3]. The main example of this kind of processes is the Lévy sheet.
\end{abstract}

Keywords : Semi-selfsimilar process, semi-stable process, IDT process.

AMS 2000 subject classification : 60G48, 60G51, 60G44, 60G10

\section{Introduction}

An $\mathbb{R}^{d}$-valued stochastic process $X=\left(X_{t}, t \geq 0\right)$ is said to be IDT if, for every $n \in \mathbb{N}$, we have

$$
\left(X_{n t}, t \geq 0\right) \stackrel{d}{=}\left(X_{t}^{(1)}+\ldots+X_{t}^{(n)}, t \geq 0\right)
$$

where $\left(X^{(1)}, t \geq 0\right), \ldots,\left(X^{(n)}, t \geq 0\right)$ are independent copies of $X$ and $\stackrel{d}{=}$ denotes equality in all finite-dimensional distributions. The notion of IDT processes has been introduced by Mansuy [3] as a generalization of Lévy processes. Various properties of IDT processes have been already investigated in [3], related for instance to their temporal self-decomposability and the characterization of IDT Gaussian processes. Regarded as a contribution to this expending topic, it is the purpose of this paper to extend some results on Lévy processes studied in [1], [2] and [4] to the case of IDT processes. In particular, we shall prove that, IDT processes are more tractable than Lévy processes, since they could be obtained by combining the selfsimilarity and strict stability. A such result is not true in general for Lévy processes. Moreover, we will prove that a necessary and sufficient condition for an IDT process to be a Lévy process is the hypothesis of independence increments. While, this condition can be circumvented when dealing with IDT processes. So it turns out that the class of IDT processes can be very rich.

The paper is organized as follows. Section 2 contains some preliminaries on stable processes and selfsimilar processes. Section 3 establishes, for IDT processes, the connection between the selfsimilar (semi-selfsimilar, resp.) processes and the strictly stable (strictly semi-stable, resp.) processes. Namely, strictly stable (strictly semi-stable, resp.) IDT process is a simple example of

*k.essebaiy@ucam.ac.ma **ouknine@ucam.ac.ma 
selfsimilar (semi-selfsimilar, resp.) process. As a byproduct, we consider the so-called Lamperti transformation for strictly semi-stable IDT processes to give a generalized semi-stable OrnsteinUhlenbeck process (see Definition 3.4).

Time-changed Lévy processes where the chronometers are more general than subordinators arise now in many fields of application, see for instance [1] and the reference therein. We shall prove (Theorem 3.6) the inheritance of IDT under time change when base processes are Lévy processes.

In section 4 we shall introduce a notion of multiparameter IDT processes and we give several examples of this kind of processes, one of them is the Lévy sheet. Contrary to the one-parameter case, we will prove that multiparameter Lévy processes are not IDT in our sense. As in the one-parameter case [3], we characterize the multiparameter IDT Gaussian processes. Moreover, we define multiparameter temporal selfdecomposable processes similar to those introduced by Barndorff-Nielsen, Meajima and Sato [1] and we prove that multiparameter IDT processes are temporal selfdecomposable.

\section{Preliminaries}

In this section we recall some definitions that we will use in the sequel. For more details the reader is referred to Sato [7].

An $\mathbb{R}^{d}$-valued random variable $X$ is called degenerate if it is a constant almost surely. An $\mathbb{R}^{d}$-valued process $\left(X_{t}, t \geq 0\right)$ is called trivial if $X_{t}$ is degenerate for every $t$.

Let $0<\alpha \leq 2$. An infinitely divisible probability measure $\mu$ on $\mathbb{R}^{d}$ is called $\alpha$-stable if, for any $a>0$, there is $\gamma_{a} \in \mathbb{R}^{d}$ such that

$$
\hat{\mu}(\theta)^{a}:=\left(\int_{\mathbb{R}^{d}} e^{i<\theta, z>} \mu(d z)\right)^{a}=\hat{\mu}\left(a^{1 / \alpha} \theta\right) e^{i<\theta, \gamma_{a}>}, \forall \theta \in \mathbb{R}^{d} .
$$

It is called strictly $\alpha$-stable if, for any $a>0$,

$$
\hat{\mu}(\theta)^{a}=\hat{\mu}\left(a^{1 / \alpha} \theta\right), \forall \theta \in \mathbb{R}^{d} .
$$

It is called $\alpha$-semi-stable if, for some $a>0$ with $a \neq 1$, there is $\gamma_{a} \in \mathbb{R}^{d}$ satisfying (1). It is called strictly $\alpha$-semi-stable if, there is some $a>0$ with $a \neq 1$ satisfying (2).

Let $\left(X_{t}, t \geq 0\right)$ be a Lévy (IDT, resp.) process on $\mathbb{R}^{d}$. It is called a $\alpha$-stable, strictly $\alpha$-stable, semi $\alpha$-stable, or strictly $\alpha$-semi-stable Lévy (IDT, resp.) process if every finite-dimensional distribution of $X$ is, respectively, $\alpha$-stable, strictly $\alpha$-stable, semi $\alpha$-stable, or strictly $\alpha$-semistable.

Let $H>0$. A stochastic process $\left(X_{t}, t \geq 0\right)$ on $\mathbb{R}^{d}$ is called $H$-selfsimilar if, for any $a>0$,

$$
\left(X_{a t}, t \geq 0\right) \stackrel{d}{=}\left(a^{H} X_{t}, t \geq 0\right) .
$$

It is called wide-sense $H$-selfsimilar if, for any $a>0$, there is a function $c(t)$ from $\mathbb{R}^{+}$to $\mathbb{R}^{d}$ such that

$$
\left(X_{a t}, t \geq 0\right) \stackrel{d}{=}\left(a^{H} X_{t}+c(t), t \geq 0\right) .
$$

It is called $H$-semi-selfsimilar if, there is some $a>0$ with $a \neq 1$ satisfying (3) and it is called wide-sense $H$-semi-selfsimilar if, for some $a>0$ with $a \neq 1$, there is a function $c(t)$ satisfying $(4)$. 


\section{Stable IDT processes}

The goal of this section is to generalize some properties of Lévy process to the case of IDT process. We first establish a link between IDT process and Lévy process than between IDT process and selfsimilar process.

Theorem 3.1. If $X=\left(X_{t}, t \geq 0\right)$ is an IDT, stochastically continuous process with independent increments, then $X$ is a Lévy process.

Proof. It suffices to prove that $X$ has stationary increments. Using the IDT property we obtain

$$
E e^{i \theta X_{k}}=\left(E e^{i \theta X_{1}}\right)^{k}, \text { for any } \mathrm{k} \in \mathbb{N} .
$$

In a similar way (5) can be obtained when $k$ is a rational time. It follows now from the stochastic continuity of $X$ that

$$
E e^{i \theta X_{t}}=\left(E e^{i \theta X_{1}}\right)^{t}, \text { for any } \mathrm{t} \in \mathbb{R}^{+} .
$$

Therefore, for any $0 \leq s<t$, we have

$$
E e^{i \theta X_{t-s}}=\left(E e^{i \theta X_{1}}\right)^{t-s}=\frac{E e^{i \theta X_{t}}}{E e^{i \theta X_{s}}}=E e^{i \theta\left(X_{t}-X_{s}\right)},
$$

where the last equality follows from the independence of increments. And since for IDT processes $X_{0}=0$ almost surely, then $X$ has stationary increments, which completes the proof.

Remark 3.1. If $\left(X_{t}, t \geq 0\right)$ is a centered Gaussian process satisfying the assumptions of the previous proposition, then $X$ is Brownian motion up to a multiplicative constant with covariance function $c(s, t)=E\left(X_{t} X_{s}\right)=(s \wedge t) c(1,1)$. In particular, for Gaussian processes, one can replace the assumption of independence of increments by stationarity of increments. Indeed, let $s<t$, since $X$ is an IDT centered Gaussian process, then it is $1 / 2$-selfsimilar (see [3]), hence

$$
E \exp \left(i\left(X_{t}-X_{s}\right)\right)=\exp \left(-\frac{1}{2}\left[(t+s) E X_{1}^{2}-2 E\left(X_{t} X_{s}\right)\right]\right) .
$$

On the other hand, we have

$$
E \exp \left(i\left(X_{t}-X_{s}\right)\right)=\left(E \exp \left(i X_{1}\right)\right)^{t-s}=\exp \left(-\frac{1}{2}(t-s) E\left(X_{1}\right)^{2}\right) .
$$

It follows from (6) and (7) that $E\left(X_{t} X_{s}\right)=s E\left(X_{1}\right)^{2}$ for $s<t$.

Proposition 3.1. Let $0<\alpha \leq 2$. A nontrivial, strictly $\alpha$-stable, $(1 / \alpha)$-selfsimilar process $\left(X_{t}, t \geq 0\right)$ is an IDT process.

Proof. Since $X$ is strictly $\alpha$-stable, we have

$$
\left(n^{(1 / \alpha)} X_{t}, t \geq 0\right) \stackrel{d}{=}\left(\sum_{i=1}^{n} X_{t}^{(i)}, t \geq 0\right), \quad \forall n \in \mathbb{N}^{*},
$$

where $X^{(1)}, \ldots, X^{(n)}$ are independent copies of $X$. On the other hand, it follows from the selfsimilarity of $X$ that

$$
\left(X_{n t}, t \geq 0\right) \stackrel{d}{=}\left(n^{(1 / \alpha)} X_{t}, t \geq 0\right)
$$

which implies $\{X(t)\}$ is an IDT process. 


\subsection{Strictly stable IDT processes}

In the case of a Lévy process $\left(X_{t}, t \geq 0\right)$, Theorem 1.4.2 in [2] prove that $\mathcal{L}\left(X_{1}\right)$ is stable if and only if $\left(X_{t}, t \geq 0\right)$ is selfsimilar. We can generalize this result as follows.

Theorem 3.2. Let $\left(X_{t}, t \geq 0\right)$ be a nontrivial, stochastically continuous, IDT process. Then $\left(X_{t}, t \geq 0\right)$ is strictly $\alpha$-stable if and only if it is $\left(\frac{1}{\alpha}\right)$-selfsimilar.

Proof. First, assume that $\left(X_{t}, t \geq 0\right)$ is $\left(\frac{1}{\alpha}\right)$-selfsimilar. Using the IDT property, we obtain

$$
\left(n^{\left(\frac{1}{\alpha}\right)} X_{t}, t \geq 0\right) \stackrel{d}{=}\left(X_{n t}, t \geq 0\right) \stackrel{d}{=}\left(\sum_{i=1}^{n} X_{t}^{(i)}, t \geq 0\right), \quad \text { for any } n \in \mathbb{N}^{*},
$$

where $X^{(1)}, \ldots, X^{(n)}$ are independent copies of $X$. Thus $\{X(t)\}$ is strictly $\alpha$-stable.

Conversely, suppose $\left(X_{t}, t \geq 0\right)$ is strictly $\alpha$-stable. Since $\{X(t)\}$ is an IDT process,

$$
\left(X_{n t}, t \geq 0\right) \stackrel{d}{=}\left(\sum_{i=1}^{n} X_{t}^{(i)}, t \geq 0\right) \stackrel{d}{=}\left(n^{\frac{1}{\alpha}} X_{t}, t \geq 0\right), \quad \text { for any } n \in \mathbb{N}^{*},
$$

and also

$$
\left(X_{\frac{t}{n}}, t \geq 0\right) \stackrel{d}{=}\left(\left(\frac{1}{n}\right)^{\frac{1}{\alpha}} X_{t}, t \geq 0\right) .
$$

Hence, for any $m \in \mathbb{N}, n \in \mathbb{N}^{*}$, we have that

$$
\left(X_{\left(\frac{m}{n} t\right)}, t \geq 0\right) \stackrel{d}{=}\left((m / n)^{\frac{1}{\alpha}} X_{t}, t \geq 0\right) .
$$

Combining this with the stochastic continuity of $\{X(t)\}$, we obtain that $\{X(t)\}$ is $\left(\frac{1}{\alpha}\right)$-selfsimilar. The proof is now complete.

Next, we will give an example of an IDT process which is not Lévy process and satisfies the above theorem.

Example 3.1. Let $S_{\alpha}$ be a strictly $\alpha$-stable random variable. The process $X$ defined by

$$
X_{t}=t^{1 / \alpha} S_{\alpha}, t \geq 0
$$

is an $(1 / \alpha)$-selfsimilar, IDT process.

Corollary 3.1. If $\alpha=2$ or $0<\alpha<1$, then $\frac{1}{\alpha}$-selfsimilar, IDT processes with stationary increments are necessarily $\alpha$-stable Lévy processes.

The proof of this corollary is straightforward from Theorem 7.5.4 in [6] and Theorem 3.2 above.

Corollary 3.2 (Sub-stable processes). Let $0<\alpha<2, \alpha<\beta \leq 2$ and $\left(Y_{t}, t \geq 0\right)$ be a symmetric $\beta$-stable IDT process and let $\xi$ be a $(\alpha / \beta)$-stable positive random variable independent of $Y$. The process $\left(X_{t}, t \geq 0\right)$ defined by

$$
X_{t}=\xi^{\frac{1}{\beta}} Y_{t},
$$

is $\left(\frac{1}{\beta}\right)$-selfsimilar, symmetric $\alpha$-stable.

Proof. The $1 / \beta$-self similarity follows from Theorem 3.2 and the symmetric $\alpha$-stability can be proved by using classical arguments on sub-stable processes (see Example 3.6.3 in [2]). So we omit the details. 


\subsection{Strictly semi-stable IDT processes}

The following result gives the connection between semi-selfsimilarity and strict semi-stability for IDT processes. The case of Lévy processes appears in [[7], Theorem 4.1].

Theorem 3.3. Let $\left(X_{t}, t \geq 0\right)$ be an $\mathbb{R}^{d}$-valued IDT, stochastically continuous process. Then 1) $\left(X_{t}, t \geq 0\right)$ is semi-stable if it is wide-sense semi-selfsimilar.

2) $\left(X_{t}, t \geq 0\right)$ is semi-selfsimilar if and only if it is strictly semi-stable.

Proof. 1) Suppose $X$ is wide-sense $H$-semi-selfsimilar, then for some $a \in(0,1) \cup(1, \infty)$, there exists a nonrandom function $c:[0, \infty) \rightarrow \mathbb{R}^{d}$, such that

$$
\left(X_{a t}, t \geq 0\right) \stackrel{d}{=}\left(a^{H} X_{t}+c(t), t \geq 0\right)
$$

Therefore, and by using IDT property, for all $t_{1}, t_{2}, \ldots, t_{m} \in \mathbb{R}_{+}$and all $\left(\theta_{1}, \ldots \theta_{m}\right) \in \mathbb{R}^{d \times m}$, we have

$$
\begin{aligned}
\left(E e^{i \sum_{k=1}^{m}\left\langle\theta_{k}, X_{t_{k}}\right\rangle}\right)^{a} & =\left(E e^{i \sum_{k=1}^{m}\left\langle\theta_{k}, X_{a t_{k}}\right\rangle}\right) \\
& =\left(E e^{i \sum_{k=1}^{m}\left\langle\theta_{k}, a^{H} X_{t_{k}}\right\rangle}\right)\left(e^{i \sum_{k=1}^{m}\left\langle\theta_{k}, c\left(t_{k}\right)\right\rangle}\right) .
\end{aligned}
$$

It remains to show that $\left(X_{t_{1}}, \ldots, X_{t_{m}}\right)$ is infinitely divisible, which follows from the IDT property. Thus $\left(X_{t}, t \geq 0\right)$ is $1 / H$-semi-stable.

2) Assume that $\left(X_{t}, t \geq 0\right)$ is strictly $\alpha$-semi-stable, then for some $a \in(0,1) \cup(1, \infty)$, we have

$$
\begin{aligned}
\left(E e^{i\left\langle\theta, a^{1 / \alpha}\left(X_{t_{1}}, \ldots, X_{t_{m}}\right)\right\rangle}\right) & =\left(E e^{i\left\langle\theta,\left(X_{t_{1}}, \ldots, X_{t_{m}}\right)\right\rangle}\right)^{a} \\
& =\left(E e^{i\left\langle\theta,\left(X_{a t_{1}}, \ldots, X_{a t_{m}}\right)\right\rangle}\right) .
\end{aligned}
$$

where the last equality follows from the IDT property. Hence $\left(X_{t}, t \geq 0\right)$ is $1 / \alpha$-semi-selfsimilar. The converse is proved in a similar way. Hence we omit the details.

Is a wide-sense $H$-selfsimilar in fact $H$-selfsimilar, if it is $H$-semi-selfsimilar? This question has an answer in the case of a stable Lévy process in [4], but we can also answer this question in the case of a stable IDT process.

Proposition 3.2. Let $\left(X_{t}, t \geq 0\right)$ be an $\mathbb{R}^{d}$-valued, nontrivial, stochastically continuous $\alpha$-stable IDT process. If it is strictly $\alpha$-semi-stable, then it is strictly $\alpha$-stable.

Proof. Assume that $\left(X_{t}, t \geq 0\right)$ is strictly $\alpha$-semi-stable, then for some $a>1$

$$
\left(X_{a t}, t \geq 0\right) \stackrel{d}{=}\left(a^{1 / \alpha} X_{t}, t \geq 0\right) .
$$

Let $t_{1}, \ldots, t_{m} \in \mathbb{R}_{+}, b_{1}, \ldots, b_{m} \in \mathbb{R}$ fixed. Then there exists a finite measure $\Gamma$ on the unit sphere $S$ of $\mathbb{R}^{d \times m}$ and a vector $\mu$ in $\mathbb{R}^{d \times m}$ with a symmetric nonnegative-definite matrix $A$, such that the characteristic function of $X:=\left(X_{t_{1}}, \ldots, X_{t_{m}}\right)$ has the following form

$$
\begin{aligned}
& E e^{i<\theta, X>} \\
& = \begin{cases}e^{\left\{-\int_{S}|<\theta, s>|^{\alpha}(1-i \operatorname{sign}(<\theta, s>) \tan (\pi \alpha / 2)) \Gamma(d s)+i<\theta, \mu>\right\}} & \text { if } \alpha \neq 1,2 \\
e^{\left\{-\int_{S}|<\theta, s>|\left(1+i \frac{2}{\pi} \operatorname{sign}(<\theta, s>) \ln |<\theta, s>|\right) \Gamma(d s)+i<\theta, \mu>\right\}} & \text { if } \alpha=1 \\
e^{\left\{-\frac{1}{2}<A \theta, \theta>+i<\theta, \mu>\right\}} & \text { if } \alpha=2 .\end{cases}
\end{aligned}
$$


The pair $(\Gamma, \mu)$ is unique, and

$$
\operatorname{sign}(x)=\left\{\begin{array}{l}
1 \quad \text { if } x>0 \\
0 \quad \text { if } x=0 \\
-1 \quad \text { if } x<0
\end{array}\right.
$$

Using the IDT property and (8), we have

$$
\left(E e^{i<\theta, X>}\right)^{a}=E e^{i<\theta,\left(X_{a t_{1}}, \ldots, X_{a t_{m}}\right)>}=E e^{i<a^{\frac{1}{\alpha} \theta, X>}}, \quad \forall \theta \in \mathbb{R}^{d \times m} .
$$

First, if $\alpha \neq 1$, then according to (9) we have $a \mu=a^{\frac{1}{\alpha}} \mu$. Which implies that $\mu=0$. Thus, $X$ is strictly $\alpha$-stable.

Let's now assume that $\alpha=1$, then

$$
\int_{S}<\theta, s>\ln |<\theta, s>| \Gamma(d s)=\int_{S}<\theta, s>\ln |<a \theta, s>| \Gamma(d s) .
$$

Consequently

$$
\int_{S} \ln (a)<\theta, s>\Gamma(d s)=0, \forall \theta \in \mathbb{R}^{d} .
$$

This means that $\int_{S} s_{k} \Gamma(d s)=0$ for $k=1,2, \ldots, d$. Which is exactly the condition for the strictly 1 -stability of $X$. Then every finite-dimensional distribution of $\left(X_{t}, t \geq 0\right)$ is strictly $\alpha$-stable. The proof is completed.

Applying the Lamperti transformation to semi-stable IDT processes, we derive a new class of periodically stationary processes. Recall that a stochastic process $\left(Y_{t}, t \in \mathbb{R}\right)$ is said to be periodically stationary with period $p(>0)$ if

$$
\left(Y_{t+p}, t \in \mathbb{R}\right) \stackrel{d}{=}\left(Y_{t}, t \in \mathbb{R}\right) .
$$

Definition 3.4. Let $0<\alpha \leq 2$ and let $\left(X_{t}, t \geq 0\right)$ be a strictly $\alpha$-semi-stable IDT, stochastically continuous process, and we define a periodically stationary process $\left(Y_{t}, t \in \mathbb{R}\right)$ by

$$
Y_{t}=e^{-t / \alpha} X_{e^{t}}
$$

We call this process a generalized $\alpha$-semi-stable Ornstein-Uhlenbeck process.

Proposition 3.3. A generalized $\alpha$-semi-stable Ornstein-Uhlenbeck process $\left(Y_{t}, t \in \mathbb{R}\right)$ is strictly $\alpha$-semi-stable.

Proof. From Theorem 3.3, point 2) we obtain

$$
\left(X_{a t}, t \geq 0\right) \stackrel{d}{=}\left(a^{1 / \alpha} X_{t}, t \geq 0\right), \quad \text { for some } a \in(0,1) \cup(1, \infty) .
$$

Combining the IDT property and (10), we have that for any $\theta=\left(\theta_{1}, \ldots, \theta_{m}\right), \theta_{k} \in \mathbb{R}^{d}, k=$ $1, \ldots, m$ and $\left(t_{1}, \ldots, t_{m}\right) \in \mathbb{R}^{m}$

$$
\begin{aligned}
\left(E e^{i\left\langle\theta,\left(Y_{t_{1}}, \ldots, Y_{t_{m}}\right)\right\rangle}\right)^{a} & =\left(E e^{i\left\langle\theta,\left(e^{-t_{1} / \alpha} X_{\left.\left.a e^{t_{1}}, \ldots, e^{-t_{m} / \alpha} X_{a e^{t_{m}}}\right)\right\rangle}\right)\right.}\right. \\
& =\left(E e^{i\left\langle\theta,\left(a^{1 / \alpha} e^{-t_{1} / \alpha} X_{\left.\left.e^{t_{1}}, \ldots, a^{1 / \alpha} e^{-t_{m} / \alpha} X_{e} t_{m}\right)\right\rangle}\right)\right.}\right. \\
& =\left(E e^{i\left\langle a^{1 / \alpha} \theta,\left(Y_{t_{1}}, \ldots, Y_{t_{m}}\right)\right\rangle}\right) .
\end{aligned}
$$

Thus $\left(Y_{t_{1}}, \ldots, Y_{t_{m}}\right)$ is strictly $\alpha$-semi-stable for any $\left(t_{1}, \ldots, t_{m}\right) \in \mathbb{R}^{m}$. The proof is completed. 


\subsection{Subordination through an IDT process}

Subordination is a transformation of a stochastic process to a new stochastic process through random time change by an increasing Lévy process (subordinator) independent of the original process. The aim of this paragraph is to investigate the case where the chronometer is an increasing IDT process .

Definition 3.5. A real-valued stochastic process $\xi=\left\{\xi_{t}, t \geq 0\right\}$ with $\xi_{0}=0$ a.s. is called a chronometer if, it is increasing, stochastically continuous.

The following result on chronometers is needed in the sequel. Its proof is obvious and so omitted.

Lemma 3.1. Let $\left(\xi_{t}^{\prime}\right)_{t \geq 0}$ be a copies of a chronometer $\xi$ then the following statements are true . $\xi_{0}^{\prime}=0$ a.s.,

- for any $t_{1}, t_{2}$ with $0 \leq t_{1} \leq t_{2}, P\left(0 \leq \xi_{t_{1}}^{\prime} \leq \xi_{t_{2}}^{\prime}\right)=1$,

- and $\xi^{\prime}$ is stochastically continuous.

The following result is inheritance of IDT under time change when base processes are Lévy processes.

Theorem 3.6. Let $X$ be a Lévy process on $\mathbb{R}^{d}$ and $\xi$ is an IDT chronometer such that $X$ and $\xi$ are independent. Then $\left(Z_{t}:=X_{\xi_{t}}: t \geq 0\right)$ is an IDT process.

Proof. Let $\xi^{(j)}, j=1, \ldots, n$ be independent copies of $\xi$. Since $X$ is independent of $\xi$, then for every $n \geq 1, \theta=\left(\theta_{1}, \ldots, \theta_{m}\right) \in\left(\mathbb{R}^{d}\right)^{m}$, we have

$$
J(n, \theta):=E \exp \left\{\sum_{k=1}^{m} i\left\langle\theta_{k}, X_{\xi_{n t_{k}}}\right\rangle\right\}=E\left[\left(E \exp \left\{\sum_{k=1}^{m} i\left\langle\theta_{k}, X_{s_{k}}\right\rangle\right\}\right)_{s_{k}=\xi_{n t_{k}}, k=1, \ldots, m}\right]
$$

By using the IDT property, we obtain

$$
J(n, \theta)=E\left[\left(E \exp \left\{\sum_{k=1}^{m} i\left\langle\theta_{k}, X_{s_{k}}\right\rangle\right\}\right)_{s_{k}=\sum_{j=1}^{n} \xi_{t_{k}}^{(j)}, k=1, \ldots, m}\right]
$$

According to the change of variables $c_{k}=\theta_{k}+\ldots+\theta_{n}$ and $t_{0}=0$, and the independence of increments of $X$, we have

$$
\begin{aligned}
J(n, \theta) & =E\left[\left(E \exp \left\{\sum_{k=1}^{m} i\left\langle c_{k}, X_{s_{k}}-X_{s_{k-1}}\right\rangle\right\}\right)_{s_{k}=\sum_{j=1}^{n} \xi_{t_{k}}^{j}, k=1, \ldots, m}\right] \\
& =E\left[\left(\prod_{k=1}^{m} E \exp \left\{i\left\langle c_{k}, X_{s_{k}}-X_{s_{k-1}}\right\rangle\right\}\right)_{s_{k}=\sum_{j=1}^{n} \xi_{t_{k}}^{(j)}, k=1, \ldots, m}\right]
\end{aligned}
$$

Now, it follows from the stationarity of the increments of $X$ and the independence of the $\xi^{(j)}$, 
$j=1, \ldots, n$, that

$$
\begin{aligned}
J(n, \theta)= & E\left[\left(\prod_{k=1}^{m} \prod_{j=1}^{n} E \exp \left\{i\left\langle c_{k}, X_{r_{k}^{j}}\right\rangle\right\}\right)_{r_{k}^{j}=\xi_{t_{k}}^{(j)}-\xi_{t_{k-1}}^{(j)}, k=1, \ldots, m, j=1, \ldots, n}\right] \\
= & E\left[\left(\prod_{j=1}^{n} E \exp \left\{\sum_{k=1}^{m} i\left\langle\theta_{k}, X_{r_{k}^{j}}\right\rangle\right\}\right)_{r_{k}^{j}=\xi_{t_{k}}^{(j)}, k=1, \ldots, m, j=1, \ldots, n}\right] \\
& =\left(E\left[\left(E \exp \left\{\sum_{k=1}^{m} i\left\langle\theta_{k}, X_{r_{k}}\right\rangle\right\}\right)_{r_{k}=\xi_{t_{k}}, k=1, \ldots, m}\right]\right)^{n} \\
& =\left(E \exp \left\{\sum_{k=1}^{m} i\left\langle\theta_{k}, X_{\xi_{t_{k}}}\right\rangle\right\}\right)^{n} .
\end{aligned}
$$

This completes the proof.

Similarly, one can prove the following result.

Proposition 3.4. Let $\left\{X_{s}: s \in \mathbb{R}_{+}^{N}\right\}$ be an $\mathbb{R}_{+}^{N}$-parameter Lévy process on $\mathbb{R}^{d}$ and let $\left\{\xi_{t}: t \geq 0\right\}$ be a $N$-dimensional subordinator in the sense of being a $N$-dimensional IDT process $\left\{\xi_{t}\right\}=$ $\left\{\left(\xi_{t}^{1}, \ldots, \xi_{t}^{N}\right)^{\top}\right\}$ that is increasing in each coordinate with the superscript $\top$ denoting the transpose, and $\left\{\xi_{t}\right\}$ independent of $\left(X(s): s \in \mathbb{R}_{+}^{N}\right)$. Define the subordinated process by composition as follows

$$
Y_{t}=X_{\xi_{t}}, \quad t \geq 0
$$

Then $\left(Y_{t}: t \geq 0\right)$ is an IDT process on $\mathbb{R}^{d}$.

\section{Multiparameter IDT processes}

In this section we introduce a notion of multiparameter infinitely divisible with respect to time (IDT) processes. A typical example of this processes is the Lévy sheet.

Definition 4.1. An $\mathbb{R}^{d}$-valued stochastic process $\left(X_{t}, t \in \mathbb{R}_{+}^{N}\right)$ is said to be IDT if for any $n=\left(n_{1}, \ldots, n_{N}\right) \in\left(\mathbb{N}^{*}\right)^{N}$,

$$
\left(X_{(n . t)}, t \in \mathbb{R}_{+}^{N}\right) \stackrel{d}{=}\left(\sum_{i=1}^{\prod_{k=1}^{N} n_{k}} X_{t}^{(i)}, t \in \mathbb{R}_{+}^{N}\right)
$$

where $X^{(1)}, \ldots, X^{\left(\prod_{k=1}^{N} n_{k}\right)}$ are independent copies of $X$ and $(n . t):=\left(n_{1} t_{1}, \ldots, n_{N} t_{N}\right)$.

In the following we give some examples of multiparameter IDT processes.

Example 4.1. 1) Let $\xi$ be a strictly $\alpha$-stable random variable, the process defined by

$$
\left(X_{t}=\left(t_{1}^{1 / \alpha} t_{2}^{1 / \alpha} \ldots . t_{N}^{1 / \alpha}\right) \xi, t \in \mathbb{R}_{+}^{N}\right),
$$


is an IDT process.

2) If $X$ is an IDT process and $\mu$ a measure on $\mathbb{R}_{+}^{N}$ such that

$$
X_{t}^{(\mu)}=\int_{\mathbb{R}_{+}^{N}} X_{(s . t)} \mu(d s), t \in \mathbb{R}_{+}^{N},
$$

is well defined, then $X^{(\mu)}$ is an IDT process.

3) Let $\left(X_{t}, t \geq 0\right)$ be an IDT process, then the multiparameter process defined by

$$
Y_{t}=X_{t_{1} t_{2} \ldots t_{N}}, \quad \text { for any } t=\left(t_{1}, \ldots, t_{N}\right) \in \mathbb{R}_{+}^{N},
$$

is IDT.

In order to show that any Lévy sheet process is IDT, we give firstly the definition of such process.

Definition 4.2. Let $\left(X_{t}, t \in \mathbb{R}_{+}^{2}\right)$ be a family of random variables on $\mathbb{R}^{d}$. we write $X_{s_{1}, s_{2}}$ instead of $X_{s}$ when $s=\left(s_{1}, s_{2}\right)^{\top}$. For $s=\left(s_{1}, s_{2}\right)^{\top}$ and $u=\left(u_{1}, u_{2}\right)^{\top}$ in $\mathbb{R}_{+}^{2}$ with $s_{1} \leq u_{1}$ and $s_{2} \leq u_{2}$, call $B=\left(s_{1}, u_{1}\right] \times\left(s_{2}, u_{2}\right]$ a rectangle in $\mathbb{R}_{+}^{2}$ and set

$$
X(B)=X_{u_{1}, u_{2}}-X_{s_{1}, u_{2}}-X_{u_{1}, s_{2}}+X_{s_{1}, s_{2}},
$$

If $B_{1}, \ldots, B_{n}$ are disjoint rectangles in $\mathbb{R}_{+}^{2}$ and $B=\cup_{j=1}^{n} B_{j}$, then set $X(B)=\sum_{j=1}^{n} X\left(B_{j}\right)$. The stochastic process $\left(X_{t}, t \in \mathbb{R}_{+}^{2}\right)$ is called a Lévy sheet if

(a) If $n \geq 2$ and $B_{1}, \ldots, B_{n}$ are disjoint rectangles, then $X\left(B_{1}\right), \ldots, X\left(B_{n}\right)$ are independent.

(b) If $B$ is a rectangle and $s \in \mathbb{R}_{+}^{2}$, then $X(B) \stackrel{d}{=} X(B+s)$.

(c) $X_{s_{1}, 0}=X_{0, s_{2}}=0$ a.s. for $s_{1}, s_{2} \in \mathbb{R}_{+}^{2}$.

(d) $X_{t} \rightarrow X_{s}$ in probability as $|t-s| \rightarrow 0$ in $\mathbb{R}_{+}^{2}$.

Proposition 4.1. Let $\left(X_{t}, t \in \mathbb{R}_{+}^{2}\right)$ be a Lévy sheet process on $\mathbb{R}^{d}$, then it is IDT.

Proof. Let $n, m \in \mathbb{N}, \theta=\left(\theta_{1}, \ldots, \theta_{p}\right) \in \mathbb{R}^{d \times p}, 0=s_{0} \leq s_{1}<\ldots<s_{p}$ and $0=t_{0}, t_{1}, \ldots, t_{p} \in \mathbb{R}_{+}$, let $\sigma$ be a permutation such that $t_{\sigma(1)} \leq \ldots \leq t_{\sigma(p)}$ and $\sigma(0)=0$. We consider disjoint rectangles $\left(B_{k}^{l}=\left(n s_{k-1}, n s_{k}\right] \times\left(m t_{\sigma(l-1)}, m t_{\sigma(l)}\right]\right), k=1, \ldots, p, l=1, \ldots, p$. Using Lévy sheet properties (see [5]), there exist a matrix $\left(c_{k}^{l}\right)_{0 \leq k, l \leq p}, c_{k}^{l} \in \mathbb{R}^{d}$ such that

$$
\begin{aligned}
E e^{i \sum_{j=1}^{p}\left\langle\theta_{j}, X_{\left(n s_{j}, m t_{j}\right)}\right\rangle} & =E e^{i \sum_{j=1}^{p}\left\langle\theta_{j}, X\left(\left(0, n s_{j}\right] \times\left(0, m t_{j}\right]\right)\right\rangle}=E e^{i \sum_{0 \leq k, l \leq p}\left\langle c_{k}^{l}, X\left(B_{k}^{l}\right)\right\rangle} . \\
& =\prod_{0 \leq k, l \leq p} E e^{i\left\langle c_{k}^{l}, X\left(B_{k}^{l}\right)\right\rangle} \\
& =\prod_{0 \leq k, l \leq p}\left(E e^{i\left\langle c_{k}^{l}, X_{1,1}\right\rangle}\right)^{\lambda\left(B_{k}^{l}\right)}=I, \quad \text { say, }
\end{aligned}
$$


where $\lambda$ is the Lebesgue measure. Since $\lambda\left(B_{k}^{l}\right)=n \times m \lambda\left(\left(s_{k-1}, s_{k}\right] \times\left(t_{\sigma(l)}, t_{\sigma(l-1)}\right]\right)$, then we have

$$
\begin{aligned}
I & =\left(\prod_{0 \leq k, l \leq p}\left(E e^{i\left\langle c_{k}^{l}, X_{1,1}\right\rangle}\right)^{\lambda\left(\left(s_{k-1}, s_{k}\right] \times\left(t_{\sigma(l)}, t_{\sigma(l-1)}\right]\right)}\right)^{n \times m} \\
& =\left(\prod_{0 \leq k, l \leq p}\left(E e^{i\left\langle c_{k}^{l}, X\left(\left(s_{k-1}, s_{k}\right] \times\left(t_{\sigma(l)}, t_{\sigma(l-1)}\right]\right)\right\rangle}\right)\right)^{n \times m} \\
& =\left(E e^{i \sum_{j=1}^{p}\left\langle\theta_{j}, X\left(\left(0, s_{j}\right] \times\left(0, t_{j}\right]\right)\right\rangle}\right)^{n \times m}
\end{aligned}
$$

This completes the proof.

Remark 4.1. If $\left(X_{t}, t \in \mathbb{R}_{+}^{N}\right)$ is an $\mathbb{R}^{d}$-valued stochastically continuous IDT process, then

$$
X_{t}=0 \text { a.s for any } t \in \mathbb{R}_{+}^{N} \text {, with } \inf _{i=1, \ldots, N} t_{i}=0 .
$$

Indeed, for any $n \geq 1, u \in \mathbb{R}^{d}$ and $t=\left(t_{1}, \ldots, t_{j-1}, 0, t_{j+1}, \ldots t_{N}\right)$, by IDT property we have

$$
E \exp \left(i\left\langle u, X_{t}\right\rangle\right)=E \exp \left(i\left\langle u, X_{\left(t_{1}, \ldots, t_{j-1}, n \times 0, t_{j+1}, \ldots, t_{N}\right)}\right\rangle\right)=\left[E \exp \left(i\left\langle u, X_{t}\right\rangle\right)\right]^{n} .
$$

Moreover the characteristic function of $X_{t}$ is non vanishing (because the laws of this variable is infinitely divisible), then

$$
E e^{i u X_{t}}=1 \text {, for all } u \in \mathbb{R}^{d} .
$$

Thus, $\mathcal{L}\left(X_{t}\right)=\delta_{0}$, where $\delta_{0}$ is the distribution concentrated at 0 and $\mathcal{L}(X)$ denotes the law of $\mathrm{X}$.

According to Mansuy [3], Proposition 1.1, any one-parameter Lévy process is IDT. The following result shows that such a result does'nt hold in the multiparameter case. We refer to Pedersen and Sato [[5], Definition 2.1] for the definition and properties of $\mathbb{R}_{+}^{N}$-parameter Lévy processes in law.

Proposition 4.2. Let $N \geq 2$. If $\left(X_{t}, t \in \mathbb{R}_{+}^{N}\right)$ is an $\mathbb{R}_{+}^{N}$-parameter Lévy processes in law satisfying the IDT property, then $X_{t}=0$ a.s, $\forall t \in \mathbb{R}_{+}^{N}$.

Proof. Assuming $X$ a such process. Then by Remark 4.1, we have

$$
E e^{i\left\langle u, X_{t}\right\rangle}=\prod_{k=1}^{N} E e^{i\left\langle u, X_{\left(0, \ldots, 0, t_{k}, 0, \ldots, 0\right)}\right\rangle}=1, \text { for any } t \in \mathbb{R}_{+}^{N}, u \in \mathbb{R}^{d} .
$$

this proves $\mathcal{L}\left(X_{t}\right)=\delta_{0}, \forall t \in \mathbb{R}_{+}^{N}$.

In the following we show that for any stochastically continuous IDT process $X$ there exists a Lévy sheet that has the same one-dimensional marginals with $X$.

Proposition 4.3. Let $\left(X_{t}, t \in \mathbb{R}_{+}^{2}\right)$ be a stochastically continuous IDT process. Then there exists a Lévy sheet process $\left(Z_{t}, t \in \mathbb{R}_{+}^{2}\right)$ such that

$$
X_{t} \stackrel{d}{=} Z_{t}, \quad \text { for any } t \in \mathbb{R}_{+}^{2} .
$$


Proof. First we note that the laws of finite dimensional marginals of an multiparameter IDT process $X$ are infinitely divisible. In particular, the law of $X_{1,1}$ is infinitely divisible, then there exists a Lévy sheet $\left(Z_{t}, t \in \mathbb{R}_{+}^{2}\right)$ with $X_{1,1} \stackrel{d}{=} Z_{1,1}$. It follows that if $Z_{t}, t \in \mathbb{R}_{+}^{2}$ is a Lévy sheet then $\mathcal{L}\left(X_{t}\right)$ is infinitely divisible and that

$$
E\left[e^{i\left\langle z, X_{t_{1}, t_{2}}\right\rangle}\right]=\left(E\left[e^{i\left\langle z, X_{1,1}\right\rangle}\right]\right)^{t_{1} t_{2}} \quad \text { for any } t_{1}, t_{2} \in \mathbb{R}_{+} .
$$

Hence

$$
X_{t} \stackrel{d}{=} Z_{t}, \quad \text { for any } t \in \mathbb{R}_{+}^{2}
$$

Definition 4.3. An stochastic process $\left(Y_{t}, t \in \mathbb{R}^{N}\right)$ is said to be strictly stationary if, for every $a \in \mathbb{R}^{N}$

$$
\left(Y_{a+t}, t \in \mathbb{R}^{N}\right) \stackrel{d}{=}\left(Y_{t}, t \in \mathbb{R}^{N}\right) .
$$

For the characterization of IDT Gaussian multiparameter processes, we will need the following

Lemma 4.1. Let $\left(Y_{t}, t \in \mathbb{R}^{N}\right)$ be a strictly stationary process, and fix $H=\left(h_{1}, \ldots, h_{N}\right) \in\left(\mathbb{R}_{+}^{*}\right)^{N}$. Define $\left(X_{t}, t \in \mathbb{R}_{+}^{N}\right)$ by

$$
X_{t}=t_{1}^{h_{1}} \ldots t_{N}^{h_{N}} Y_{\left(\log \left(t_{1}\right), \ldots, \log \left(t_{N}\right)\right)}, t \in\left(\mathbb{R}_{+}\right)^{N} ; X_{0}=0,
$$

then $\left(X_{t}, t \in \mathbb{R}_{+}^{N}\right)$ is $H$-selfsimilar in the following sense

$$
\left(X_{(a . t)}, t \in\left(\mathbb{R}_{+}\right)^{N}\right) \stackrel{d}{=}\left(a_{1}^{h_{1}} \ldots a_{N}^{h_{N}} X_{t}, t \in\left(\mathbb{R}_{+}\right)^{N}\right) \text { for any } a \in\left(\mathbb{R}_{+}^{*}\right)^{N} .
$$

Conversely, if $\left(X_{t}, t \in\left(\mathbb{R}_{+}\right)^{N}\right)$ is $H$-selfsimilar process, then its Lamperti transform

$$
\left(Y_{t}:=e^{\left\{-\sum_{k=1}^{N} h_{k} t_{k}\right\}} X_{\left(e^{t_{1}}, \ldots, e^{t_{N}}\right)}, t \in \mathbb{R}^{N}\right),
$$

is strictly stationary.

Proof. Assume that $\left(Y_{t}, t \in \mathbb{R}^{N}\right)$ is strictly stationary, then for any $a=\left(a_{1}, \ldots, a_{N}\right) \in\left(\mathbb{R}_{+}^{*}\right)^{N}$, we have

$$
\begin{aligned}
\left(X_{(a . t)}, t \in \mathbb{R}_{+}^{N}\right) & =\left(\left(a_{1} t_{1}\right)^{h_{1}} \ldots\left(a_{N} t_{N}\right)^{h_{N}} Y_{\left(\log \left(a_{1}\right)+\log \left(t_{1}\right), \ldots, \log \left(a_{N}\right)+\log \left(t_{N}\right)\right)}, t \in \mathbb{R}_{+}^{N}\right) \\
& \stackrel{d}{=}\left(\left(a_{1} t_{1}\right)^{h_{1}} \ldots\left(a_{N} t_{N}\right)^{h_{N}} Y_{\left(\log \left(t_{1}\right), \ldots, \log \left(t_{N}\right)\right)}, t \in \mathbb{R}_{+}^{N}\right) \\
& =\left(a_{1}^{h_{1}} \ldots a_{N}^{h_{N}} X_{t}, t \in \mathbb{R}_{+}^{N}\right),
\end{aligned}
$$

thus, $\left(X_{t}, t \in \mathbb{R}_{+}^{N}\right)$ is $H$-selfsimilar. Conversely, since $\left(X_{t}, t \in \mathbb{R}^{N}\right)$ is $H$-selfsimilar, for any $b=\left(b_{1}, \ldots, b_{N}\right) \in \mathbb{R}_{+}{ }^{N}$ we have

$$
\begin{aligned}
& \left(Y_{(t+b)}, t \in \mathbb{R}^{N}\right)=\left(e^{\left\{-\sum_{k=1}^{N} h_{k}\left(t_{k}+b_{k}\right)\right\}} X_{\left(e^{t_{1}+b_{1}}, \ldots, e^{\left.t_{N}+b_{N}\right)}\right.}, t \in \mathbb{R}^{N}\right) \\
& \stackrel{d}{=}\left(e^{\left\{-\sum_{k=1}^{N} h_{k} t_{k}\right\}} X_{\left(e^{t_{1}}, \ldots, e^{t_{N}}\right)}, t \in \mathbb{R}^{N}\right)=\left(Y_{t}, t \in \mathbb{R}^{N}\right) \text {. }
\end{aligned}
$$

Thus, $\left(Y_{t}, t \in \mathbb{R}^{N}\right)$ is strictly stationary. 
Proposition 4.4. Let $\left(X_{t}, t \in \mathbb{R}_{+}^{N}\right)$ be a stochastically continuous, centered Gaussian process. Then the following properties are equivalent:

i) $\left(X_{t}, t \in \mathbb{R}_{+}^{N}\right)$ is an IDT process.

ii) The covariance function $c(s, t):=E\left(X_{s} X_{t}\right),(s, t) \in \mathbb{R}_{+}^{N} \times \mathbb{R}_{+}^{N}$, satisfies

$$
c(\alpha . s, \alpha . t)=\alpha_{1} \ldots \alpha_{N} c(s, t), \text { for any } \alpha \in\left(R_{+}^{*}\right)^{N} .
$$

iii) The process $\left(X_{t}, t \in \mathbb{R}_{+}^{N}\right)$ is $\left(\frac{1}{2}, \ldots, \frac{1}{2}\right)$-selfsimilar.

iv) $\left(Y_{y}:=e^{\left\{-\sum_{k=1}^{N} h_{k} y_{k}\right\}} X_{\left(e^{y_{1}}, \ldots, e^{y_{N}}\right)}, y \in \mathbb{R}^{N}\right)$ is strictly stationary.

Proof. $i) \Leftrightarrow i i)$. We have, $\left(X_{t}, t \in \mathbb{R}_{+}^{N}\right)$ is IDT if and only if, for any $n=\left(n_{1}, \ldots, n_{N}\right) \in \mathbb{N}^{N}, s, t \in$ $\mathbb{R}_{+}^{N}$

$$
c(n . s, n . t)=n_{1} \ldots n_{N} c(s, t),
$$

and also, for any $q=\left(q_{1}, \ldots, q_{N}\right) \in \mathbb{Q}_{+}^{N}, s, t \in \mathbb{R}_{+}^{N}$

$$
c(q . s, q . t)=q_{1} \ldots q_{N} c(s, t) .
$$

Moreover, since $X$ is stochastically continuous, its covariance function is continuous. Hence, using the density of $\mathbb{Q}_{+}$in $\mathbb{R}_{+}$, we obtain the result.

ii) $\Leftrightarrow$ iii). Since the centered Gaussian process is characterized by its covariance function, we obtain the result easily.

$i i i) \Leftrightarrow i v)$. It is a direct application of Lemma 4.1 for $H=(1 / 2, \ldots, 1 / 2)$.

Example 4.2. Let $\left(B_{t}, t \in \mathbb{R}_{+}^{N}\right)$ ba a Brownian sheet. i.e. the centered, real-valued Gaussian random field with covariance function $E(B(t) B(s))=\prod_{i=1}^{N} s_{i} \wedge t_{i}$. Since its covariance function satisfies the point ii) of Proposition 4.4 , then $\left(B_{t}, t \in \mathbb{R}_{+}^{N}\right)$ is an IDT process.

We will define the multiparameter temporally selfdecomposable processes which extend the one introduced in [1] and relate this notion with the IDT processes.

Definition 4.4. An $\mathbb{R}^{d}$-valued stochastic process $X=\left(X_{t}, t \in \mathbb{R}_{+}^{N}\right)$ is temporally selfdecomposable if, for every $c \in(0,1)^{N}$, there exist two independent processes $X^{(c)}=\left(X_{t}^{(c)}, t \in \mathbb{R}_{+}^{N}\right)$, and $U^{(c)}=\left(U_{t}^{(c)}, t \in \mathbb{R}_{+}^{N}\right)$ on $\mathbb{R}^{d}$, such that

$$
X \stackrel{d}{=} X^{(c)}+U^{(c)}
$$

where $X^{(c)} \stackrel{d}{=}\left(X_{(c . t)}, t \in \mathbb{R}_{+}^{N}\right)$ and $U^{c}$ is called the c-residual of $X$.

For every $m \geq 2$, we say that $X$ is temporally selfdecomposable of order $m$ if, it is temporally selfdecomposable and for any $c \in(0,1)^{N}$, the c-residual process of $X$ is temporally selfdecomposable of order $(m-1)$. When $X$ is temporally selfdecomposable of order $m$ for all $m$, we call it infinitely temporally selfdecomposable.

Proposition 4.5. An $\mathbb{R}^{d}$-valued, stochastically continuous IDT process is infinitely temporally selfdecomposable. 
Proof. By stochastic continuity and IDT property, we have for any $\left(t_{1}, \ldots, t_{m}\right) \in\left(\mathbb{R}_{+}^{N}\right)^{m}, \xi \in \mathbb{R}^{m}$ and $c=\left(c_{1}, \ldots, c_{N}\right) \in(0,1)^{N}$

$$
\begin{aligned}
E e^{\sum_{j=1}^{m} i\left\langle\xi_{j}, X_{t_{j}}\right\rangle} & =\left(E e^{\sum_{j=1}^{m} i\left\langle\xi_{j}, X_{\left(c_{1} t_{j}^{1}, \ldots, c_{N} t_{j}^{N}\right)}\right\rangle}\right)^{1 / \prod_{k=1}^{N} c_{k}} \\
& =\left(E e^{\sum_{j=1}^{m} i\left\langle\xi_{j}, X_{c . t_{j}}\right\rangle}\right)\left(E e^{\sum_{j=1}^{m} i\left\langle\xi_{j}, X_{\left(c_{1}\left(\frac{1}{\prod_{k=1}^{N} c_{k}}-1\right) t_{j}^{1}, \ldots, c_{N}\left(\frac{1}{\prod_{k=1}^{N} c_{k}}-1\right) t_{j}^{N}\right)}\right\rangle}\right) .
\end{aligned}
$$

Therefore $\left(X_{t}, t \in \mathbb{R}_{+}^{N}\right)$ is temporally selfdecomposable and

$$
\left(X_{t}, t \in \mathbb{R}_{+}^{N}\right) \stackrel{d}{=}\left(X_{c . t}+U_{t}^{c}, t \in \mathbb{R}_{+}^{N}\right),
$$

where $U^{c}$ is independent of $\left(X_{c . t}, t \in \mathbb{R}_{+}^{N}\right)$ and

$$
\left(U_{t}^{c}, t \in \mathbb{R}_{+}^{N}\right) \stackrel{d}{=}\left(X_{\left(c_{1}\left(\frac{1}{\Pi_{k=1}^{N} c_{k}}-1\right) t^{1}, \ldots, c_{N}\left(\frac{1}{\prod_{k=1}^{N} c_{k}}-1\right) t^{N}\right)}, t \in \mathbb{R}_{+}^{N}\right)
$$

It follows from (11) that $U^{c}$ is stochastically continuous and IDT. The same steps as above applied to $U^{c}$, proves that $U^{c}$ is temporally selfdecomposable and its residuel process is stochastically continuous and IDT. Continuing in exactly the same manner, we conclude that $X$ is infinitely temporally selfdecomposable.

\section{References}

[1] Barndorff-Nielsen, O.E., Meajima, M. and Sato, K. (2005) Infinite divisibility for stochastic processes and time change, to appear in Journal of Theoretical Probability.

[2] Embrechts, P. And Meajima, M. (2002) Selfsimilar Processes, Princeton University Press, William Sreet, Princeton, New Jersey 08540.

[3] Mansuy, R. (2005) On processes which are infinitely divisible with respect to time, arXiv:math $/ 0504408$.

[4] Meajima, M. And Sato, K. (1999) Semi-selfsimilar processes, J. Theoret. Probab. 12, 347-373.

[5] Pedersen, J. and Sato, K. (2004) Semigroups and processes with parameter in a cone, Abstract and Applied analysis: pp. 499-513.

[6] Samorodnitsky, G. and Taqqu, M.S. (1994) Stable Non-Gaussian processes, Chapman and Hall, London.

[7] Sato, K. (1999) Lévy processes and Infinitely Divisible Distributions, Cambridge Univ. Press, Cambridge. 